\title{
Research and implementation of image feature point matching method based on OpenCV
}

\author{
Rong Liu, Danmei Peng, Yang Liu \\ Faculty of Geomatics, East China University of Technology, Nanchang, 321000, China \\ email:rliu@ecit.edu.cn
}

Keywords: OpenCV; Feature point; SIFT; BRISK; Image matching

\begin{abstract}
This paper described the distance metric of the Euclidean distance of SIFT operator and Hamming distance of BRISK operator represented based on OpenCV to extract and descript feature points on the two relatively low altitude remote sensing images, and then chose BruteForceMatch (violent match) and FlannBasedMatch (approximate Closest match) two matching methods to match in the same matching operator. Lastly, the RANSAC algorithm was chose to estimate the fundamental matrix, eliminating the effects of error matching on the fundamental matrix accuracy and stability. By using extreme geometric constraints to reject the mistake matching points in error matching, and improve the robustness and accuracy of the matching. The results show that the performance of FlannBasedMatch method is more advantageous.
\end{abstract}

\section{Introduction}

In recent years, the rapid development of geographic information science and technology and related industries, which bring great benefits to the various sectors of society. However, the requirements of remote sensing data are more and more, and getting the currency and timely requirements for high-resolution remote sensing imagery and ground fine spatial information are also increasing. But for small areas and large-scale mapping, conventional aerial systems because of the high costs, low performance, subject to aircraft transitions strict conditions and other factors are can not meet the large-scale remote sensing images quickly update needs. With the development of the UAV and auxiliary equipment, low altitude remote sensing meets the needs of this aspect, quickly becoming the focus of widespread concern. Image matching as a key technology in the processing of low altitude remote sensing data, the matching quality will directly affect the subsequent results. The essence of image matching is to find out the corresponding image points in two images and the relationship between two images, then according to the real situation of the reference space, connecting same scene or object in image under the different point of view.

This paper mainly studied the gray-scale variation obvious feature point on the image. There are many feature point matching operators,it described the distance metric of the Euclidean distance of SIFT operator and Hamming distance of BRISK operator represented based on OpenCV to extract and descript feature points on the two relatively low altitude remote sensing images, and then chose BruteForceMatch (violent match) and FlannBasedMatch (approximate Closest match) two matching methods to match in the same matching operator. Lastly, the RANSAC algorithm was chose to estimate the fundamental matrix, eliminating the effects of error matching on the fundamental matrix accuracy and stability. By using extreme geometric constraints to reject the mistake matching points in error matching, and improve the robustness and accuracy of the matching.

\section{Feature point extraction and matching}

\section{(1)Feature point extraction}

This paper unified selected BRISK algorithm to rapidly extract the feature points, and then used the feature points which extracted by SIFT and BRISK algorithm to describe, generate their own descriptors. 
SIFT is an algorithm based on local features of images, it was proposed by Lowe in 1999 and was summarized in 2004. Experiments shown that, SIFT algorithm for changes in light intensity and solid angle can maintain a certain stability, and because on the whole and within the scale space can be well localized that it can reduce the noise problem. The main steps of the image local feature extraction algorithm into feature point detection scale space, the precise allocation feature point, the feature point direction and creating a feature point descriptor. This paper used the approach of SIFT algorithm to generate feature point descriptor. Compared with the SIFT algorithm, BRISK algorithm have more advantages on high matching accuracy or computational speed, it can get real-time processing of video frames. BRISK algorithm consists of feature points extracted by the FAST and generating binary descriptors.

BRISK algorithm was based on the scale space formed by FAST feature point and its own image plane where the use of FAST score to find the maximum value, and then utilizing FAST 9-16 to calculate its score. As shown in Figure 1. In the circumference 16 pixels within at least nine pixels is greater than or less than the center pixel $p+\tau$ or $p-\tau$. Where $\tau$ is the set threshold. These points are the pre-selected key points. For any of the pixels at circumference, its status $\mathrm{S}$ compares to the center pixel $\mathrm{p}$ is represented as one of the following state.

$$
S_{p \rightarrow x}=\left\{\begin{array}{lc}
d, & \left(I_{p \rightarrow x} \leq I_{p}-\tau\right) \\
s, & \left(I_{p}-\tau<I_{p \rightarrow x}<I_{p}+\tau\right) \\
b, & \left(I_{p}+\tau \leq I_{p \rightarrow x}\right)
\end{array}\right.
$$

Thus, the 16 pixels at circumference can be divided into three sets, the central pixel FAST score is calculated as formula 2.

$$
V=\max \left(\sum_{x \in S}\left|I_{p \rightarrow x}-I_{p}\right|-\tau, \sum_{x \in S}\left|I_{p}-I_{p \rightarrow x}\right|-\tau\right)
$$

Firstly, in each floor of scale space used the same threshold doing FAST9-16 detecting, determining a region of interest ROI. Secondly, scale space non-maxima suppression is used in a point of the region. Lastly, we could search for feature points. If the finding point met following two conditions was considered as feature point.1. The FAST score of that point was more than the around eight neighborhood points in its image layer; 2.The FAST scores of that point score greater than all the points in two layers adjacent area.

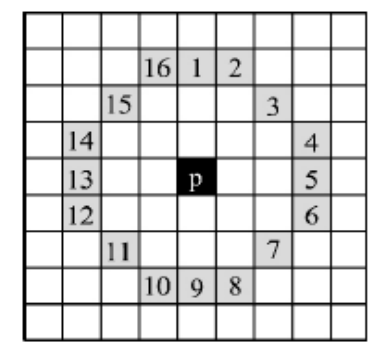

\section{(2)Matching method}

Fig.1 The key point detection model of FAST 9：16

This paper introduced BruteForceMatch method which selected different distance metric according to different spatial scales for absolute matching between the data sets descriptor. Concrete steps can be understood as: the original data set generated a descriptor $q_{\text {to calculate each distance }}$ between all of the descriptors which generated in training set, so the minimum distance of feature descriptor $t$ is the descriptor $q$ matching objects. Compared with BruteForceMatch method, FlannBasedMatch method used different distance metric calculating the similar distance between the data set descriptor based on different spatial scales. FLANN is a library that can be used in a high-dimensional space fast nearest neighbor matching, which includes almost all of the methods we used to search for the nearest match. What's more the library can be automatically selected what closest approach to search for matching data sets according to the obtained experimental data set. At the same time, this method used a decision tree structure instead of a linear search way of the BruteForceMatch method, so that the search time was shorter. 


\section{Experimental examples}

This experimental used the BRISK operator to extract feature points. BRISK operators as an improved sub-FAST extraction algorithm, with better rotational invariance, scale invariance, and robustness. It also has the characteristic of FAST extraction that can fast extract feature points.

Descriptors were selected to the SIFT operator and BRISK operator.

This article configured OpenCV on Visual Studio 2012, and then used the C ++ implementation BRISK + SIFT and BRISK + BRISK combination of image feature point extraction and description. We were selected BruteForceMatcher and FlannBasedMatcher for the same combination two matching methods to compare.

The paper selected two adjacent sequence images to compose stereoscopic image low altitude remote sensing image. As shown in figure 2. Firstly, BRISK + SIFT combination were selected BruteForceMatch and FlannBasedMatch two matching methods to match and contrast. Then set a certain threshold to be filtered matching results. Finally selecting findFundamentalMat function on OpenCV with the parameter of the function is set to FM_RANSAC algorithm to eliminate mismatches, improving the matching robustness and precision. The matching results and contrast performances are shown in Figure 3 and Table 1. FlannBasedMatch method uses much less time than BruteForceMatch method in matching, and the quantity of extracting feature points is almost equal after threshold processing and fundamental matrix filtering, satisfying the matching requirements.
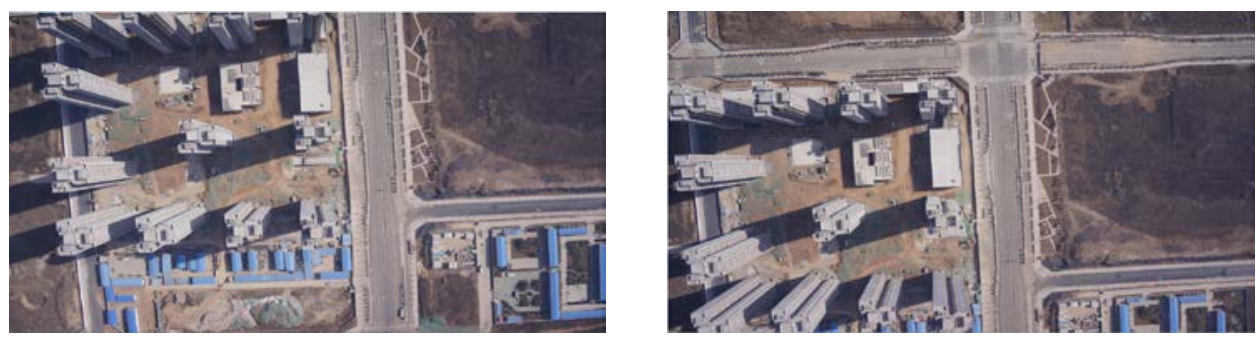

Fig.2 Stereo image
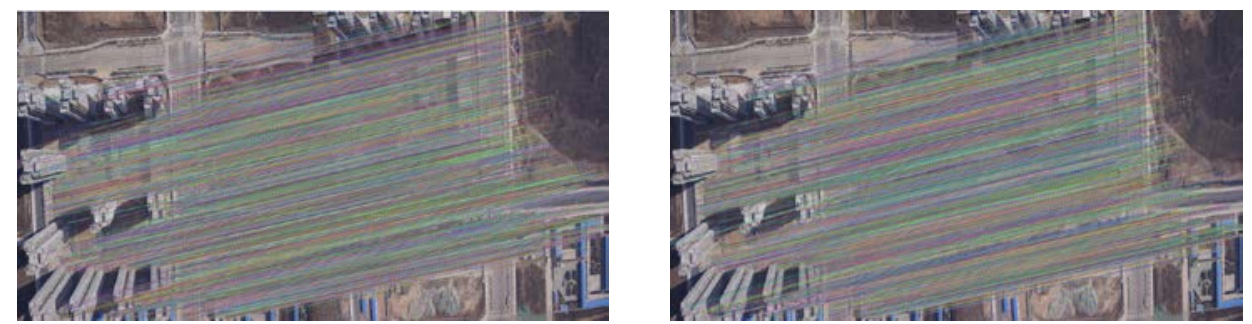

Fig.3 The matching results of BruteForceMatch and FlannBasedMatch

Table 1 The performances of two matching methods

\begin{tabular}{ccc}
\hline & $\begin{array}{c}\text { BRISK+BRISK } \\
\text { +BruteForceMatch }\end{array}$ & $\begin{array}{c}\text { BRISK+BRISK+FlannBase } \\
\text { dMatch }\end{array}$ \\
\hline $\begin{array}{c}\text { Extracting points } \\
\text { Time to generate } \\
\text { descriptors } \\
\text { Matching Time }\end{array}$ & 68600/65534 & $\begin{array}{l}68600 / 65534 \\
13353 \mathrm{~ms}\end{array}$ \\
$\begin{array}{c}\text { Threshold } \\
\text { extracting points } \\
\text { After filtration }\end{array}$ & 12972 & $98062 \mathrm{~ms}$ \\
mismatching points & 8690 & 13692 \\
\hline
\end{tabular}




\section{Conclusion}

This paper described two different matching methods respectively to two types of distance matching operator of Euclidean distance and Hamming distance measurement for feature matching, which is based on the OpenCV. The results showed that no matter which kind of matching operator, FlannBasedMatch method matching time much less than BruteForceMatch matching method, and after threshold processed and filtered, the quantity of extracted feature points almost equal. It is suggested that choosing FlannBasedMatch matching method in the process of feature points matching. However, this article does not compare the performances of SIFT operator and BRISK operator in the same matching method, and the problem of SIFT operator to generate descriptors time is too slow not resolved either, it remains to be further studied.

\section{Acknowledgement}

In this paper, the research was sponsored by the Foundation of Key laboratory of watershed ecology and geographical environment monitoring, National Administration of Surveying, Mapping and Geoinformation (Project No. 201512026).

\section{References}

[1] Ke Tao. The application of SIFT features in the automatic matching for low altitude aerial images[J].Science of Surveying and Mapping ， 2009， 34 (4) :23-26.

[2] Cai Jing. Feature Point Extraction and Matching Algorithm in Aerophotogrammetry[J].Journal of Beijing Institute of Clothing Technology，2012，44-53.

[3] Hei Haiqing. Study on Fast and Automatic Images Matching for Low Altitude Remote Sensing[J].Journal of East China Institute of Technology，2014，37（4） : 433-437.

[4] Lowe DG. Distinctive image features from scale-invariant key points, international Journal of Computer Vision, 2004, 60(2): $91-110$.

[5] Wang Song. SIFT based image matching algorithm research[D].Xidian University, 2013.

[6] Wei Xing. Rapid matching and transform algorithm of images based on the Electronic Measurement Technology, 2015，38 (3) :43-47.

[7] Du Jun. On Characteristics of BRISK Image Registration Extraction and Description [J]. Journal of Shiyan Technical Institute ， 2012， 25(1):106-109.

[8] Marius Muja and David G. Lowe. Scalable Nearest Neighbor Algorithms for High

Data. Pattern Analysis and Machine Intelligence (PAMI), 2014, 36. 